\title{
Immunoblastic morphology as a possible prognostic indicator for the outcome of the patients with diffuse large $B$ cell lymphoma in era of the rituximab based treatment: single centre experience
}

\author{
S. Trajkova ${ }^{1}$, I. Panovska-Stavridis ${ }^{1}$, A. Stojanovic ${ }^{1}$, G. Petrusevska ${ }^{2}$, D. Dukovski ${ }^{1}$, M. \\ Ivanovski $^{1}$, A. Pivkova-Veljanovska ${ }^{1}$, M. Popova-Simjanovska ${ }^{1}$, L. Cadievski ${ }^{1}$, L. Cevreska ${ }^{1}$
}

${ }^{1}$ University Clinic for Hematology, Skopje, R. Macedonia

${ }^{2}$ Institute for Pathology, Ss.Cyril and Methodius University, Faculty of Medicine, Skopje, R. Macedonia

Received: 7 March 2013

Accepted: 25 March 2013

*Correspondence:

Dr. Sanja Trajkova,

E-mail: sanjatrajkovamd@yahoo.com

(C) 2013 Trajkova $\mathrm{S}$ et al. This is an open-access article distributed under the terms of the Creative Commons Attribution License, which permits unrestricted use, distribution, and reproduction in any medium, provided the original work is properly cited.

\begin{abstract}
Recently the results from one large prospective study indicated that immunoblastic morphology and not immunohistohemical features predict the outcome of the Diffuse large B lymphoma (DLBL). In order to investigate the prediction value of the immunoblastic morphology (IB) as a possible prognostic indicator for the outcome of our DLBL patient treated with the Rituximab (R)-CHOP regimen we conducted a retrospective study. Our study enrolled 192 DLBL patients diagnosed and treated at the University Clinic of Hematology in the period between February 2002 and December 2007. They were all treated with R-CHOP regimen and the median follow-up of the patient was 36 months. We analyzed the biopsy samples immunohistochemically for markers of germinal center (BCL6), postgerminal center (MUM1) and apoptosis (BCL2).The patients were categorized as DLBL(132; 68.7\%), IB(60; 31.2). The median overall survival time (OS) were 59.3 months in DLBL group and 42.2 months in IB group, and time to treatment (TT) were 56.8 and 30.6 months respectively for the IB group. The DLBL and IB groups were comparable regarding the age, gender distributions and all others already established prognostic parameters as performance status, advanced IPI, albumin level except for the low IPI 0-2 which was statistically associated with the DLBL group $(p=.024)$. Our results did not show any statistical survival advantage and better outcome for the patient classified as DLBL when treated with R-CHOP and indicate that immunohistohemical markers do not really reflect the molecular diversity of the tumor. Our work shows that IB morphology is a major risk factor in DLBL patients treated with R$\mathrm{CHOP}$. Therefore this morphology appears to capture some adverse molecular events that a currently hard to detect with routine diagnostic procedures.
\end{abstract}

Keywords: DLBL, Immunoblastic morphology, Rituximab, Prognosis

\section{INTRODUCTION}

Diffuse large B-cell lymphoma (DLBCL) is the most common lymphoma subtype characterized with aggressive clinical course that comprises a heterogeneous group of lymphomas in terms of morphology, immunophenotype and molecular abnormalities. ${ }^{1}$ Currently, a standard first line treatment for this disease is immunochemotherapy, combination of the antiCD20 antibody rituximab with anthracycline based chemotherapy regimen.

International Prognostic Index (IPI) and its development is a milestone in the analysis of the heterogeneity of prognosis in patients with diffuse large B-cell lymphoma. IPI enables patients to be assigned to several clinical risk 
groups and for treatment strategies to be stratified accordingly. A major step forward was the segregation of DLBCL into distinct subtypes based on gene expression profiling. Two main subgroups were identified that correspond to different stages of lymphocyte development: the germinal center B-cell (GCB)-like type showed a gene expression profile reminiscent of normal germinal center B cells, whereas the activated B-cell (ABC)-like type had a gene signature similar to that of activated peripheral blood $\mathrm{B}$ cells. A third intermediate group could not be allocated to either GCB or ABC type. The different gene expression patterns corresponded to different clinical outcomes. Several attempts have been made to substitute this gene expression-based classification by more routinely applicable methods. ${ }^{2}$ This classification can be used to stratify chemotherapytreated patients into low- and high-risk groups and it has prognostic significance in response to chemotherapy, as the patients with the germinal center signature have a better survival compared with the activated B-cell signature. As immunohistochemical classification of cell of origin would be more practical than gene expressionbased methods, several attempts have been performed to identify the germinal center and non- germinal center subgroups of DLBCL by immunohistochemistry (IHC). ${ }^{2}$ The most widely applied Hans algorithm based on immunostaining for B-cell lymphoma (BCL6), interferon regulatory factor-4/multiple myeloma-1(IRF4/MUM1), cluster of differentiation $\mathrm{CD} 10$, reproduces the gene expression-based classification, with a result of $87 \%$ for the GCB and $73 \%$ for the non-GCB phenotypes with a misclassification rate of $20 \% .^{3}$ According to same studies, immunohistochemically defined germinal center patients have significantly better survival than the nongerminal center patients ${ }^{3}$, when the patients are treated only with chemotherapy, whereas in other studies no differences in the survival rates have been seen in patients treated with immunochemotherapy, where rituximab is added to standard chemotherapy regiment $\mathrm{CHOP}^{4,5}$

Further, recent results, obtained in the immunochemotherapy settings are inconsistent. Some studies found no significant correlation between the cell of origin and outcome in the patients treated with immunochemotherapy.,

Recently the results from one large prospective study published in Blood (2010), revealed the data from the RICOVER-60 trial of the German group-DSHNHL and indicated that immunoblastic morphology and not immunohistohemical features predict the outcome of the DLBL. ${ }^{8}$

In order to investigate the prediction value of the immunoblastic morphology (IB) as a possible prognostic indicator for the outcome of our DLBL patient treated with the Rituximab (R)-CHOP regimen we conducted a retrospective study.

\section{METHODS}

\section{Patients and treatments}

Our study enrolled 192 DLBL patients diagnosed and treated at the University Clinic of Hematology, Skopje, Macedonia in the period between February 2002 and December 2007. They were all with CD20-positive aggressive BCLs and were assigned to 8 cycles of RCHOP (cyclophosphamide, doxorubicin, vincristine, and prednisolone) regimen. The median follow-up of the patient was 36 months. The study was conducted in accordance with the Helsinki declaration. The baseline clinical characteristics were collected and the risk stratification scoring performed applying the International Prognostic Index. ${ }^{9}$

\section{Immunohistochemical stainings}

Immunohistochemistry was performed at Institute of Pathology, Medical Faculty, Skopje, Macedonia. All tissue biopsies were fixed in $10 \%$ buffered formalin and were routinely processed and embedded in paraffin and 4-6 micm sections were cut and stain in hematoxylin and eosin (H\&E). Immunohistochemistry was performed on 4-6 micm sections by using a streptavidin-biotin complex technique. The IHC panel consisted of antibodies against CD20, BCL2, BCL6, MUM1. The primary antibodies, suppliers, and staining conditions are listed in Table 1. Subtyping followed the definitions of 2008 WHO classification. $^{10}$ Immunoreactivity was determined without any knowledge of the clinical data of the patients. Antigen expression was evaluated by assessing the percentage of immunoreactive tumor cells in $10 \%$ increments. The cases were subclassified by using the modified algorithm described by Hans et al (Figure 1). ${ }^{3}$ For evaluation of BCL2, BCL6, MUM1 the staining was interpreted as positive if $30 \%$ or more of the tumor cells showed expression. Using the modified Hans algorithm the cases were assigned to immunoblastic group if BCL6 or MUM1 were positive or both MUM1 and BCL6 were positive, and if BCL2 alone or both MUM1 and BCL2 and BCL6 were positive the cases were assigned to DLBCL group.

Table 1: Antibodies and methods used in IHC stains.

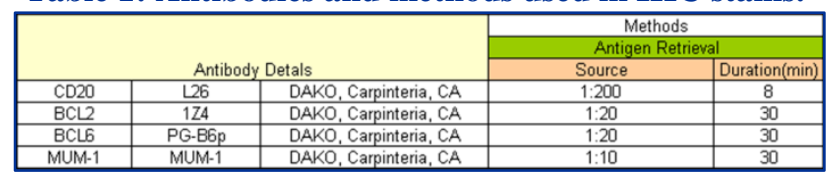

\section{Statistical analyses}

For statistical analysis we used $\mathrm{X}^{2}$ test to compare characteristics of two subgroups of patients, mean age was compared with t-test. Overall survival was defined as the time from initial diagnosis to the date of death or last contact. Time to treatment failure was defined as the time from initial diagnosis to the date of disease progression, treatment discontinuation, or patient death. The log-rank 
test was used to compare the survival distributions. OS and TTF were estimated according to Kaplan- Meier. P values less than 0.05 were considered statistically significant. The analysis was conducted with Statistic7 for Window.

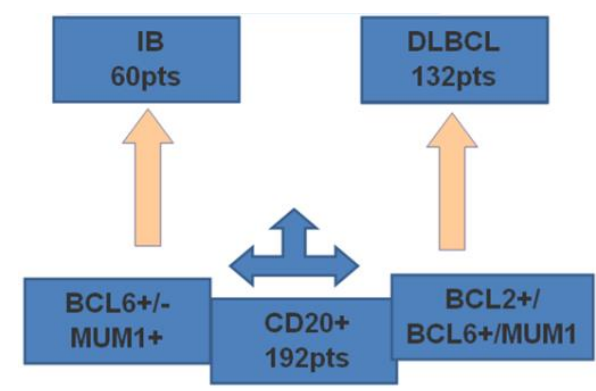

Figure 1: Modified Hans IHC classifier.

\section{RESULTS}

\section{Patient characteristics}

Table 2 provides an overview regarding the clinical parameters of all 192 patients with DLBL (60 patients with tumor classified as Immunoblastic (IB) DLBL, and 132 patients with tumor classified as DLBL). The two groups were statistically comparable regarding the age, gender distributions and all others already established prognostic parameters as performance status, advanced IPI, albumin level except for the low IPI 0-2 which was statistically associated with the GCB group $(p=.024)$ (Figure 2). As can be seen $100 \%$ of tumor samples expressed CD20, 20.8\% of the IB group were BCL2 positive, and $81 \%$ of DLBCL were positive for the same marker, $97.2 \%$ of the IB group were BCL6 positive, and 52.2 of were DLBCL positive for BCL6, $98 \%$ of the IB group were MUM1 positive, and $39 \%$ of DLBCL were positive for the same marker Immunohistochemical profile is presented on Figure 3.

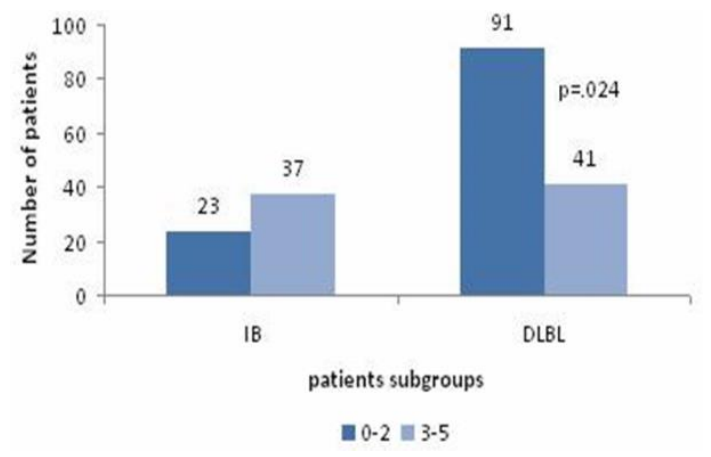

Figure 2: Distribution of patients according to IPI score.

Survival of patients with DLBL: The median overall survival time (OS) were 42.2 months in IB group and 59.3 months in DLBL group, and time to treatment (TTF) were 30.6 and 56.8 months respectively for the both groups (Figure 4, 5). Seventy percent of patient with DLBL group were alive in the end of the study, and $53.3 \%$ with IB immunophenotype respectively. Patient statistical evidence is presented on Figure 6.

Table 2: Clinical features of DLBCL patients.

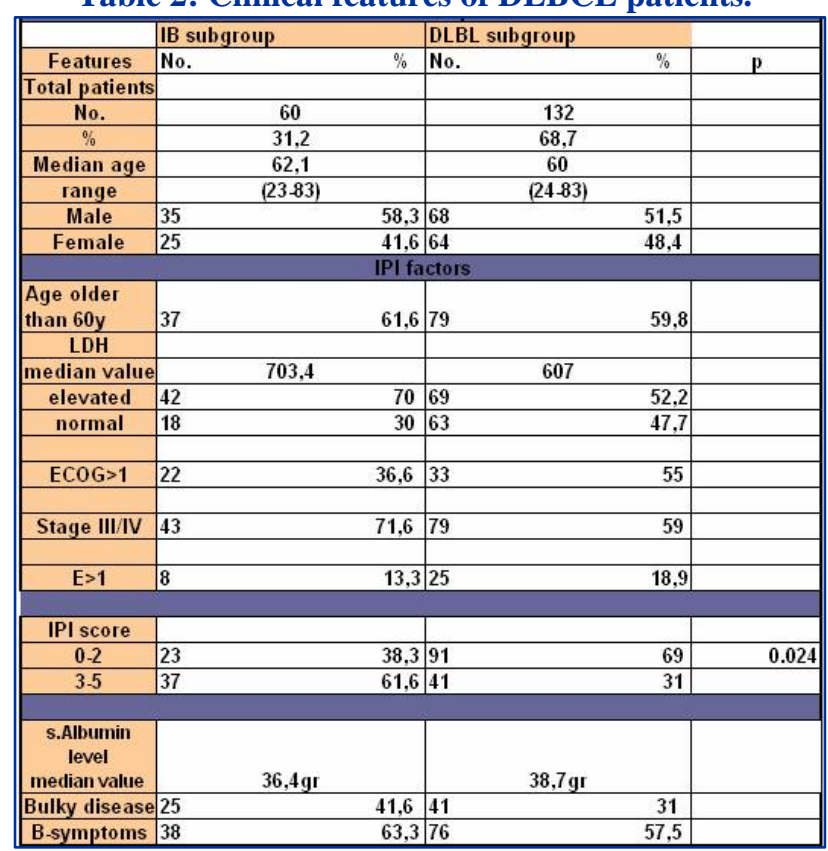

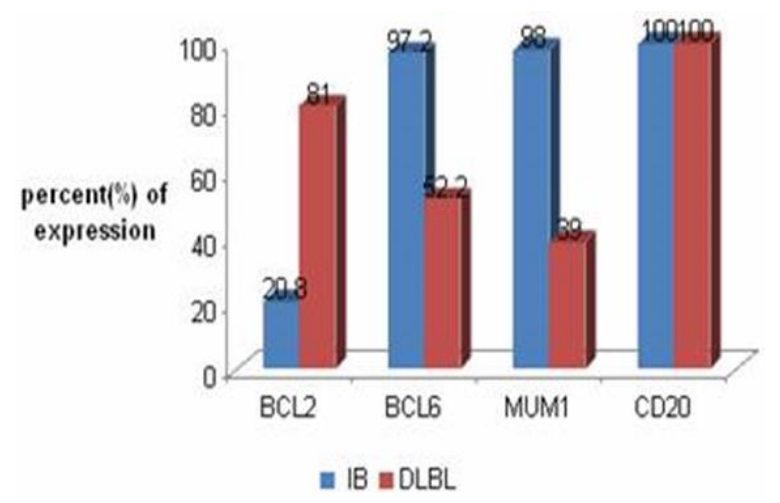

Figure 3: Distribution according to IHC parameters.

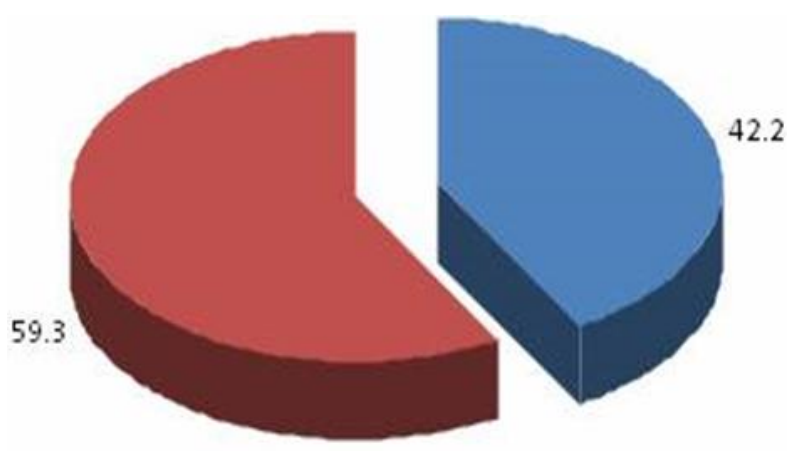

- $O S I B=O S D L B L$

Figure 4: Distribution of patients according to Overall survival time (months). 


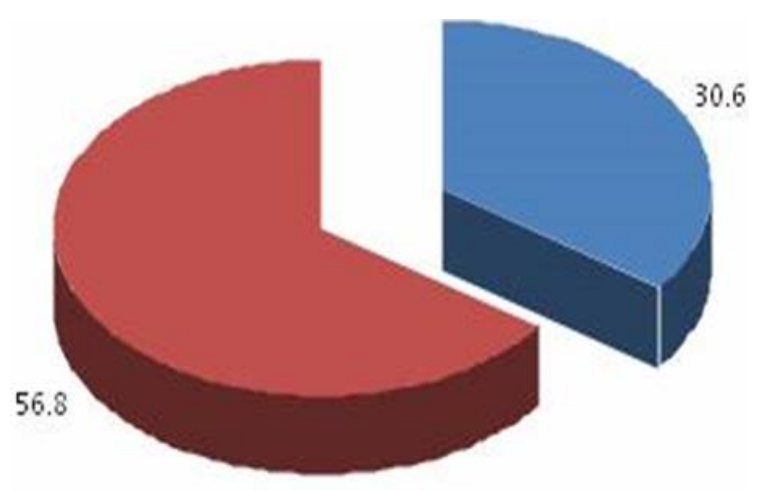

- TTF IB $=$ TTF DLBL

Figure 5: Distribution of patients according to median time to treatment failure (months).

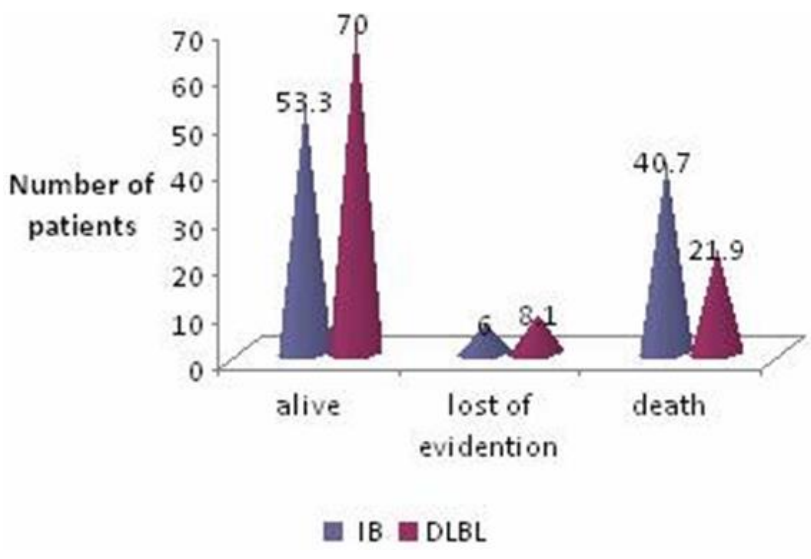

Figure 6: Distribution of patient's evidence.

\section{DISCUSSION}

The aim of this study was to investigate the prediction value of the immunoblastic morphology (IB) as a possible prognostic indicator for the outcome of our DLBL patient treated with the Rituximab (R)-CHOP regimen.

The immunoblastic morphology as an important risk factor has historic features, it has been described by Lennert a long time ago, and its negative prognostic impact of IB morphology in DLBCL has been recognized in 1997, 2002 in Non Hodgkin Lymphoma Classification Project.

In our study 192 patients were treated with 8 cycles of CHOP chemotherapy with rituximab. IB morphology of the tumor cells emerged as a significant adverse prognostic factor that retained its prognostic relevance even when the clinical IPI factors were included. The prognostic impact of IB morphology was prominent within the patients cohort treated with RCHOP. The widely used Hans classifier ${ }^{3}$ based on CD10, BCL6, MUM1 assigns DLBCL into two subcategories, as germinal center B-cell Like (GCB) and non-GCB DLBL, failed to show prognostic significance in the patients treated with rituximab.

The gene expression-based distinction between GCB and non-GCB DLBL carried a prognostic impact in the CHOP treatment era. The IHC algorithms were developed to translate the complex and multigene expression in to more applicable standards to routine formalin-fixed, paraffin-embedded tumor tissues; results from retrospective analyses are contradictory. In the CHOP treatment era, Hans classifier, or modified version of it, predicted survival of DLBCL patients in some studied $^{3,11-14}$, but not in others. ${ }^{15,16}$ But the results were contradictory in DLBCL patients treated with R-CHOP protocol. ${ }^{14,17,18}$

Ott et al worked on this problem in line with previous reports on a negative prognostic impact of IB morphology, like large prospective DLBCL trial from CHOP treatment era-NHL-B1 and B2 by the DSHNHL. ${ }^{19}$ They now proved the data that IB morphology carries important prognostic information but in R-CHOP treatment era. In some series IB morphology represent a negative prognostic factor. ${ }^{20}$

The most widely used antigens in DLBCL are of minor value when it comes to risk assessment of DLBCL patients treated with R-CHOP. The expression levels of individual biomarkers by tumor cells such as BCL-2 and BCL-6 in line with previous studies ${ }^{21,22}$, also provide important prognostic information in DLBCL. Different studies have yielded conflicting and inconclusive results, which probably reflect the heterogeneity of the disease and the various methods used. Overexpression of BCL-2 is a significant prognostic factor in non GCB lymphoma, more specifically we have found that rituximab overcome the adverse influence of BCL-2 overexpression on survival in non GCB DLBCL.

In the study published by Bernd et al for the German High Grade Non-Hodgkin's Lymphoma Study Group (DSHNHL) $^{19}$, they correlated the morphological subtype and the immunohistochemical classifier proposed by Hans et al, and they found a significantly higher number of non-GCB type cases within the immunoblastic group than in the centroblastic group. However, since this correlation was not very strong, morphology apparently cannot be regarded as an adequate surrogate for immunophenotype, although it was obvious that both, morphology and immunophenotype are indicators of distinct tumor biology. This concept is also supported by gene expression studies in which a similar correlation between immunoblastic morphology and non-GCB molecular profile has been observed.

Bernd et $\mathrm{al}^{19}$ for the German High Grade Non-Hodgkin's Lymphoma Study Group (DSHNHL) found that intensified therapy led to a significant improvement of the outcome in comparison to that obtained with the standard CHOP regimen. This effect is not, however, 
likely to be sufficient to explain why they could not confirm the prognostic value of the Hans classifier or other previously reported immunohistochemical markers.

The combination of the CHOP regimen with the monoclonal anti-CD20 antibody rituximab (R-CHOP) has considerably improved the outcome of patients with DLBCL. In this context, there is also some evidence to indicate that the impact of prognostic markers may be altered with the use of R-CHOP. The effect of rituximab does not, however, completely abolish the biological attributes of these lymphomas since at least some of the markers, for instance MHC class II gene expression, retain their prognostic value although the observable effects may be diminished.

Therefore, this study is suitable for identifying novel prognostic factor due to the high rate of events and concomitant high power. We presented our results on data sets including patients treated with rituximab with immunoblastic morphology with inferior survival comparing with other DLBL patients. Our study suggests in part new markers and that morphological subgrouping can be indicative of prognosis independently of IPI factors.

\section{REFERENCES}

1. Swerdlow SH, Campo E, Harris NL, et al. WHO Classification of Tumors of Hematopoietic and Lymphoid Tissues. IARCH Press: Lyon, 2008.

2. Alizadeh AA, Eisen MB, Davis RE, et al. Distinct types of diffuse large B-cell lymphoma identified by gene expression profiling. Nature 2000;403:503-11.

3. Hans CP, Weisenburger DD, Greiner TC, et al. Confirmation of the molecular classification of diffuse large B-cell lymphoma by immunohistochemistry using a tissue microarray. Blood 2004;103:275-82.

4. Colomo L, Lopez-Guillermo A, Perales M, et al. Clinical impact of the differentiation profile assessed by immunophenotyping in patients with diffuse large B-cell lymphoma. Blood 2003;101:7884.

5. Paepe P, Achten R, Verhoef G, et al. Large cleaved and immunoblastic lymphoma may represent two distinct clinicopathologic entities within the group of diffuse large B-cell lymphomas. J Clin Oncol 2005;23:7060-8.

6. Nyman H, Adde M, Karjalainen-Lindsberg ML, et al. Prognostic impact of immunohistochemically defined germinal center phenotype in diffuse large B-cell lymphoma patients treated with immunochemotherapy. Blood 2007;109:4930-5.

7. Wilson WH, Dunleavy K, Pittaluga S, et al. Phase II study of dose-adjusted EPOCH and rituximab in untreated diffuse large B-cell lymphoma with analysis of germinal center and post-germinal center biomarkers. J Clin Oncol 2008;26:2717-24.
8. Ott G, Ziepert M, Klapper W, et al. Immunoblastic morphology but not the immunohistochemical GCB/nonGCB classifier predicts outcome in diffuse large B-cell lymphoma in the RICOVER-60 trial of the DSHNHL. Blood 2010;116:4916-25.

9. A predictive model for aggressive non-Hodgkin's lymphoma. The International Non-Hodgkin's Lymphoma Prognostic Factors Project. N Engl J Med 1993;329:987-94.

10. Swerdlow SH, Campo E, Harris NL, et al. World Health Organization of Tumors of Hematopoietic and Lymphoid Tissues. Lyon, France: IARC Press;2008.

11. Berglund M, Thunberg U, Amini RM, et al. Evaluation of immunophenotype in diffuse large Bcell lymphoma and its impact on prognosis. Mod Pathol. 2005;18:1113-20.

12. Haarer CF, Roberts RA, Frutiger YM, et al. Immunohistochemical classification of de novo, transformed, and relapsed diffuse large B-cell lymphoma into germinal center B-cell and nongerminal center B-cell subtypes correlates with gene expression profile and patient survival. Arch Pathol Lab Med 2006;130:1819-24.

13. Muris JJ, Meijer CJ, Vos W, et al. Immunohistochemical profiling based on Bcl-2, CD10 and MUM1 expression improves risk stratification in patients with primary nodal diffuse large B cell lymphoma. J Pathol 2006;208:714-23.

14. Nyman H, Adde M, Karjalainen-Lindsberg ML, et al. Prognostic impact of immunohistochemically defined germinal center phenotype in diffuse large B-cell lymphoma patients treated with immunochemotherapy. Blood 2007;109:4930-5.

15. Natkunam Y, Farinha P, Hsi ED, et al. LMO2 protein expression predicts survival in patients with diffuse large B-cell lymphoma treated with anthracycline- based chemotherapy with and without rituximab. J Clin Oncol 2008;26:447-54.

16. Veelken H, Vik DS, Schulte Moenting J, et al. Immunophenotype as prognostic factor for diffuse large B-cell lymphoma in patients undergoing clinical risk-adapted therapy. Ann Oncol 2007;18:931-9.

17. Fu K, Weisenburger DD, Choi WW, et al. Addition of rituximab to standard chemotherapy improves the survival of both the germinal center B-cell-like and non-germinal center B-cell-like subtypes of diffuse large B-cell lymphoma. J Clin Oncol 2008;26:458794.

18. Seki R, Ohshima K, Fujisaki T, et al. Prognostic impact of immunohistochemical biomarkers in diffuse large B-cell lymphoma in the rituximab era. Cancer Sci 2009;100:1842-7.

19. Bernd HW, Ziepert M, Thorns C, et al. Loss of HLA-DR expression and immunoblastic morphology predict adverse outcome in diffuse large B-cell lymphoma - analyses of cases from two prospective randomized clinical trials. Haematologica 2009;94:1569-80. 
20. Diebold J, Anderson JR, Armitage JO, et al. Diffuse large B-cell lymphoma: a clinicopathologic analysis of 444 cases classified according to the updated Kiel classification. Leuk Lymphoma 2002;43:97-104.

21. Mounier N, Briere J, Gisselbrecht C, et al. Rituximab plus CHOP(R-CHOP) overcomes bcl-2associated resistance to chemotherapy in elderly patients with diffuse large $\mathrm{B}$ cell lymphoma (DLBCL). Blood 2003;101:4279-84.

22. Winter JN, Weller EA, Homing S, et al. Prognostic significance of Bcl-6 protein expression in DLBCL treated with $\mathrm{CHOP}$ or R-CHOP: a prospective correlative study. Blood 2006;107:4207-13.

DOI: 10.5455/2320-6012.ijrms20130501

Cite this article as: Trajkova $\mathrm{S}$, Panovska-Stavridis I, Stojanovic A, Petrusevska G, Dukovski D, Ivanovski M, Pivkova-Veljanovska A, Popova-Simjanovska M, Cadievski L, Cevreska L. Immunoblastic morphology as a possible prognostic indicator for the outcome of the patients with diffuse large B cell lymphoma in era of the rituximab based treatment: single centre experience. Int J Res Med Sci 2013;1:47-52. 